\title{
Atomic Self-ordering in Heteroepitaxially Grown Semiconductor Quantum Dots due to Relaxation of External Lattice Mismatch Strains
}

\author{
Peter Möck ${ }^{1}$, Teya Topuria ${ }^{1}$, Nigel D. Browning ${ }^{1}$, Robin J. Nicholas ${ }^{2}$, and Roger G. Booker ${ }^{3}$ \\ ${ }^{1}$ Department of Physics (MC 273), University of Illinois at Chicago, 845 W. Taylor Street, Chicago, IL 60607- \\ 7059, U.S.A; *pmoeck@uic.edu \\ ${ }^{2}$ Department of Physics, Clarendon Laboratory, University of Oxford, Parks Road, Oxford, OX1 3PU, U.K. \\ ${ }^{3}$ Department of Materials, University of Oxford, Parks Road, Oxford, OX1 3PH, U.K.
}

\section{ABSTRACT}

Thermodynamic arguments are presented for the formation of atomic order in heteroepitaxially grown semiconductor quantum dots. From thermodynamics several significant properties of these systems can be derived, such as an enhanced critical temperature of the disorder-order transition, the possible co-existence of differently ordered domains of varying size and orientation, the possible existence of structures that have not been observed before in semiconductors, the occurrence of atomic order over time, and the occurrence of short range order when the growth proceeds at low temperatures. Transmission electron microscopy results support these predictions. Finally, we speculate on the cause for the observed increase in life time of (In,Ga)As/GaAs quantum dot lasers [H-Y. Liu et al., Appl. Phys. Lett. 79, 2868 (2001)].

\section{INTRODUCTION}

The growth of self-assembled semiconductor quantum dots (QDs) by the heteroepitaxial StranskiKrastanow mode and its variants has developed rapidly over recent years [1]. Regardless of the deposition parameters, alloying of the elements or compounds (e.g. the formation of $(\mathrm{Ge}, \mathrm{Si}$ ) [2], (In, Ga)As [3], $(\mathrm{Cd}, \mathrm{Zn}) \mathrm{Se}$ [4], ect) that constitute the QD system is known to take place during the growth. This alloying is even considered to be crucial for the Stranski-Krastanow growth mode to operate in semiconductors [5]. Since the deposition and alloying are random events, these QDs will have the structure of the prototype of the constituents of the alloy, i.e. the atoms will (initially) be randomly distributed.

A tacit assumption throughout the quantum dot community is that Stranski-Krastanow grown random alloy QDs are structurally stable. Recent experimental evidence, however, indicates that this is for several III-V and II-VI compound semiconductor systems not the case [6-8]. In these systems it appears that it is atomically ordered or phase separated QDs that are structurally stable, i.e. the initial random alloy transforms to a partially ordered superlattice structure. In hind sight, it is surprising that structural transformations by means of atomic ordering and phase separation within Stranski-Krastanow grown QDs have so far not been taken into account, as there is a large body of experimental and theoretical literature which describes such transformations in heteroepitaxial semiconductor alloy layers, e.g. [10], [11], and review [12]. As these effects lead to both a reduction of the stored lattice mismatch energy and a lower band gap, such ordering should in principle lead to QDs with enhanced properties.

In this paper, we will advance qualitative thermodynamic arguments in favor of atomic ordering in heteroepitaxially grown QDs. From these arguments we will predict a significant increase in the critical temperature for the disorder-order transition, the co-existence of differently ordered domains of varying size and orientation, and the possible existence of structures that have not been observed before in semiconductors. Furthermore, these arguments also predict that long- and short-range atomic ordering occurs over time, even at room temperature, and that short-range order may occur when the growth proceeds at low temperatures. All of these predictions are borne out by experimental observations [6-8]. Representative transmission electron microscopy (TEM) images and diffraction patterns are presented here to support these predictions. Using thermodynamic arguments, we will speculate on the cause of the observed increase in the life time of (In,Ga)As/GaAs quantum dot lasers [13].

N8.8.1 


\section{THERMODYNAMICAL ARGUMENTS}

In thermodynamic terms, any structural transformation in a crystal can be explained by the minimization of the Gibbs free energy

$$
\mathrm{G}=\mathrm{E}-\mathrm{T} \mathrm{S}+\mathrm{p} \mathrm{V}
$$

where $\mathrm{E}$ is the internal energy, $\mathrm{T}$ the absolute temperature, $\mathrm{S}$ the entropy, $\mathrm{p}$ the pressure, and $\mathrm{V}$ the volume of the crystal. The requirement of a smaller band gap in the quantum dot than in the surrounding matrix is usually obtained for solid solutions with larger lattice constants. This means that heteroepitaxially grown QDs are typically compressively strained to a few percent and we will refer to them below as ordinarily strained quantum dots (OS-QDs) and use the subscript os. Assuming that OSQDs are completely embedded in a matrix with a smaller lattice constant but the same structural prototype, the product of the hydrostatic pressure on them (due to external lattice mismatch stresses) and their volume can be approximated by the product of their bulk modulus (B) and the change in their volume $(\Delta \mathrm{V})$,

$$
\mathrm{p} \mathrm{V}=\mathrm{B}_{\text {os }} \Delta \mathrm{V}_{\mathrm{os}}
$$

Now, we consider a transformation from an OS-QD with the sphalerite structure into an atomically ordered quantum dot (subscript ao) with a different structural prototype that is negligibly strained, i.e. $\Delta \mathrm{V}_{\text {aо }} \sim 0$, and where the contribution of the product of the atmospheric pressure and $\mathrm{V}$ to the Gibbs free energy is also neglected). This type of transformation is observed experimentally [6-8], (Figs. 1 and 2). Combining the energy minimization principle, (1), and (2) we obtain for this kind of structural transformation

$$
\mathrm{E}_{\mathrm{os}}-\mathrm{T} \mathrm{S}_{\mathrm{os}}+\mathrm{B}_{\mathrm{os}} \Delta \mathrm{V}_{\mathrm{os}}>\mathrm{E}_{\mathrm{ao}}-\mathrm{T} \mathrm{S}_{\mathrm{ao}}
$$

Solving for the critical temperature, $\mathrm{T}_{\mathrm{c}}$, for the disorder-order transition where both phases are in equilibrium, we obtain

$$
\mathrm{T}_{\mathrm{c}}=\frac{E_{o s}-E_{a o}+B_{o s} \cdot \Delta V_{o s}}{S_{o s}-S_{a o}}
$$

As $T_{c}$ (and any other $T$ ) is positive, and $S_{o s}-S_{a o}$ is also considered to be positive, the numerator in (4) has to be positive as well. For a sufficiently large product $\mathrm{B}_{\mathrm{os}} \Delta \mathrm{V}_{\mathrm{os}}$, this condition can be met by both possibilities $\mathrm{E}_{\mathrm{os}}>\mathrm{E}_{\mathrm{ao}}$ or $\mathrm{E}_{\mathrm{os}}<\mathrm{E}_{\mathrm{ao}}$. In either case, the larger the product $\mathrm{B}_{\mathrm{os}} \Delta \mathrm{V}_{\mathrm{os}}$ is, i.e. the effect of the external lattice mismatch stress, the larger $T_{c}$ will be. The Gibbs-Helmholtz equation (1) and the energy minimization principle tell us only which structural transformations will eventually happen, but not how fast such transformations will happen and how high the obtainable degree of order will be.

Such information can be derived qualitatively for an enhanced $\mathrm{T}_{\mathrm{c}}$ from the classical theories of long[14] and short-range [15] atomic ordering as cooperative phenomena. These theories state that at any $\mathrm{T}$ below $T_{c}$ the equilibrium degree of order will be the larger the higher $T_{c}$ is. In order to approach the equilibrium degree of order at any $\mathrm{T}$ below $\mathrm{T}_{\mathrm{c}}$, thermal treatments can be performed at this particular temperature. If $\mathrm{T}_{\mathrm{c}}$ is enhanced, a higher thermal treatment temperature can be chosen and the approach to equilibrium will be faster. On cooling below $T_{c}$, partial order will initially be quickly established in the vicinity of $T_{c}$ but complete order will only be achieved at $T=0$. Although the order parameter for long range order drops to zero at $\mathrm{T}_{\mathrm{c}}$ [14], short range order exists above the critical temperature to an appreciable amount [15] and may act as seeds for the formation of long range order.

Finally, quenched-in vacancies should play an important role in the atomic rearrangement processes in OS-QDs. In strain gradients that exist in the surroundings of OS-QDs, vacancies will move preferentially to places of higher compressive strain [16]. Provided that the kinetic energy of the vacancies is large enough to pass the tensile strain barrier that surrounds the OS-QDs, they will end up inside these entities and facilitate local atomic rearrangements. The quotient of the number of vacancies $\left(n_{V}\right)$ to the number of atoms $(\mathrm{N})$ in a crystal is given by 


$$
\frac{n_{V}}{N}=\exp \frac{-F_{V}}{k \cdot T}
$$

where $\mathrm{F}_{\mathrm{V}}$ is the formation energy of a vacancy and $\mathrm{k}$ is Boltzmann's constant. Assuming, for example, $\mathrm{F}_{\mathrm{V}}$ $\sim 1 \mathrm{eV}$, the effect of a fast quench from a growth temperature of $500{ }^{\circ} \mathrm{C}$ to room temperature $(300 \mathrm{~K})$ may be an up to ten orders of magnitude higher vacancy concentration (i.e. exp $\left(8.614 \times 10^{-5} \times 300\right)^{-1}-\exp$ $\left.\left(8.614 \times 10^{-5} \times 773.15\right)^{-1}\right)$ than in the same system in thermodynamic equilibrium. Provided that ordinarily strained quantum dots are capable of attracting a sizable amount of these excess vacancies, thermodynamics driven atomic rearrangement processes will speed up significantly.

\section{THEORETICAL IMPLICATIONS AND EXPERIMENTAL SUPPORT}

To demonstrate the structural instability of heteroepitaxially grown OS-QDs, we will estimate the values of the terms in (3) and (4) for one particular example, i.e. an $\operatorname{In}_{0.75} \mathrm{Ga}_{0.25} \mathrm{P}$ QD embedded in a $\mathrm{Ga}_{0.25} \mathrm{In}_{0.75} \mathrm{P}$ matrix. Since the material parameters in (3) and (4) are the same order of magnitude for other semiconductor systems, other OS-QDs should follow the same trends. To extrapolate between the respective values for InP and GaP, we will employ Vegard's law. Although an order of magnitude estimation would be sufficient to support the qualitative reasoning, we will use accurate values where available. Based on the elastic constants given in ref. [17], the bulk modulus of $\operatorname{In}_{0.75} \mathrm{Ga}_{0.25} \mathrm{P}$ (in the sphalerite structure) is $75.4 \mathrm{GPa}$. Also from [17], the lattice constant of an unstrained $\mathrm{In}_{0.75} \mathrm{Ga}_{0.25} \mathrm{P}$ quantum dot with the sphalerite structure is $0.5764 \mathrm{~nm}$ and the lattice constant of the unstrained surrounding $\mathrm{Ga}_{0.75} \mathrm{In}_{0.25} \mathrm{P}$ matrix is $0.5555 \mathrm{~nm}$. This results in an external lattice misfit strain of $\sim 3.6 \%$, which we assume to be evenly distributed between the QD and the immediate surrounding matrix. We will assume typical dimensions for an unstrained QD as $25 \mathrm{~nm}$ (length) $\times 25 \mathrm{~nm}$ (width) $\times 4 \mathrm{~nm}$ (height). Assuming that half the strain, i.e. $1.8 \%$, is accommodated by the QD, we obtain a volume of $\sim 2367.4$ $\mathrm{nm}^{3}$ and an absolute volume change of $\sim 132.6 \mathrm{~nm}^{3}$. With the strain evenly distributed between the OSQD and the matrix, the coherent $\operatorname{In}_{0.75} \mathrm{Ga}_{0.25} \mathrm{P}$ quantum dot (with sphalerite structure) has a lattice constant of $0.56595 \mathrm{~nm}$. This results in $\sim 13.06 \times 10^{3}$ unit cells per quantum dot and with 8 atoms per unit cell, the QD contains $\sim 104.48 \times 10^{3}$ atoms.

Calculations based on density functional perturbation theory obtained a value of $18.3 \mathrm{meV}$ for the internal energy per atom of unstrained $\mathrm{In}_{0.75} \mathrm{Ga}_{0.25} \mathrm{P}$ with sphalerite structure and an internal energy of 11.8 meV for unstrained $\operatorname{In}_{0.75} \mathrm{Ga}_{0.25} \mathrm{P}$ with famatinite structure [10], (space group $\mathrm{I} \overline{4} 2 \mathrm{~m}$ with 16 atoms per unit cell resulting from cation ordering into a superlattice [12]). From

$$
\frac{\delta S}{\delta T}=\frac{C_{p}}{T}
$$

and the specific heat at constant pressure $\mathrm{C}_{\mathrm{p}}=0.28+10^{-4} \mathrm{~T}\left[\mathrm{~J} \mathrm{~g}^{-1} \mathrm{~K}^{-1}\right]$ between 298 and $910 \mathrm{~K}$ for InP in the sphalerite structure [18], we obtain by integration

$$
\mathrm{S}(\mathrm{T})=0.28 \ln \mathrm{T}+10^{-4} \mathrm{~T} \quad\left[\mathrm{~J} \mathrm{~g}^{-1} \mathrm{~K}^{-1}\right]
$$

We assume (for lack of more specific data and the fact that the entropy of mixing [19] contributes only $2.4 \mathrm{eV} \mathrm{K}^{-1}$ to the total entropy when $25 \%$ of the In atoms are replaced on random positions by $\mathrm{Ga}$ atoms) that this relation is also valid for the OS-QD of $\operatorname{In}_{0.75} \mathrm{Ga}_{0.25} \mathrm{P}$. The entropy for the atomically ordered QD is assumed to be half that of the OS-QD. With a relative molar weight of $\operatorname{In}_{0.75} \mathrm{Ga}_{0.25} \mathrm{P}$ of $134.515 \mathrm{~g} \mathrm{~mol}^{-1}$ and Advogadro's constant, we obtain at $\mathrm{T}=300 \mathrm{~K}$ the following entropies: $\mathrm{S}_{\mathrm{os}} \sim 237 \mathrm{eV} \mathrm{K}^{-1}$ and $\mathrm{S}_{\mathrm{ao}} \sim 118.5$ $\mathrm{eV} \mathrm{K}^{-1}$.

Equation (3) gives now for $\mathrm{T}=300 \mathrm{~K}$ the structural stability determining inequality $(18.3 \times 104.48-$ $237 \times 300) \mathrm{eV}+6.241 \times 10^{4} \mathrm{eV}>(11.8 \times 104.48-118.5 \times 300) \mathrm{eV}$. Resolved and divided by the number of atoms in the QD, we obtain the inequality: $-0.66^{\mathrm{eV}} /$ atom $+0.6^{\mathrm{eV}} /$ atom $>-0.33^{\mathrm{eV}} /$ atom. These inequalities mean that without the external lattice mismatch strain, the sphalerite structure would possess the lower Gibbs free energy and be stable at room temperature (as observed in unstrained bulk crystals), but with 
the estimated contribution of the lattice mismatch strain on the OS-QD, the energy balance is reversed and the unstrained atomically ordered superlattice is the preferred structure of the system. While one may argue that our value for $S_{a o}$ (and, thus, the right hand side of these inequalities) appears to be somewhat arbitrary, it is obvious that the external lattice mismatch strain contributes significantly to the Gibbs free energy of the OS-QD (on the left hand side of these inequalities) and, therefore, destabilizes it. Using the same values and approximations, we obtain from (4) an estimation of the critical temperature

$$
\mathrm{T}_{\mathrm{c}}=\left(\frac{9.3234}{0.28 \cdot \ln T_{c}+T_{c} \cdot 10^{-4}}+\frac{8.5678 \cdot 10^{2}}{0.28 \cdot \ln T_{c}+T_{c} \cdot 10^{-4}}\right) \mathrm{K}
$$

for the reversible transformation between the two possible structure of an $\operatorname{In}_{0.75} \mathrm{Ga}_{0.25} \mathrm{P}$ QD a value of $~$ $486 \mathrm{~K}$. The second term in (8) is due to the effect of the external lattice mismatch strain and obviously enhances $\mathrm{T}_{\mathrm{c}}$ significantly. However, using the same values as above, if all of the external lattice mismatch strain is accommodated by the OS-QDs alone and the lattice of the matrix remains undistorted, $\mathrm{T}_{\mathrm{c}} \sim 852$ $\mathrm{K}$, i.e. a temperature within the range that is commonly used to growth (In,Ga)P quantum dots [20]. An enhancement of $\mathrm{T}_{\mathrm{c}}$ of this kind has been observed to take place in Stranski-Krastanow grown (In, Ga)Sb quantum dots in GaSb matrix, as shown in Figure 1.
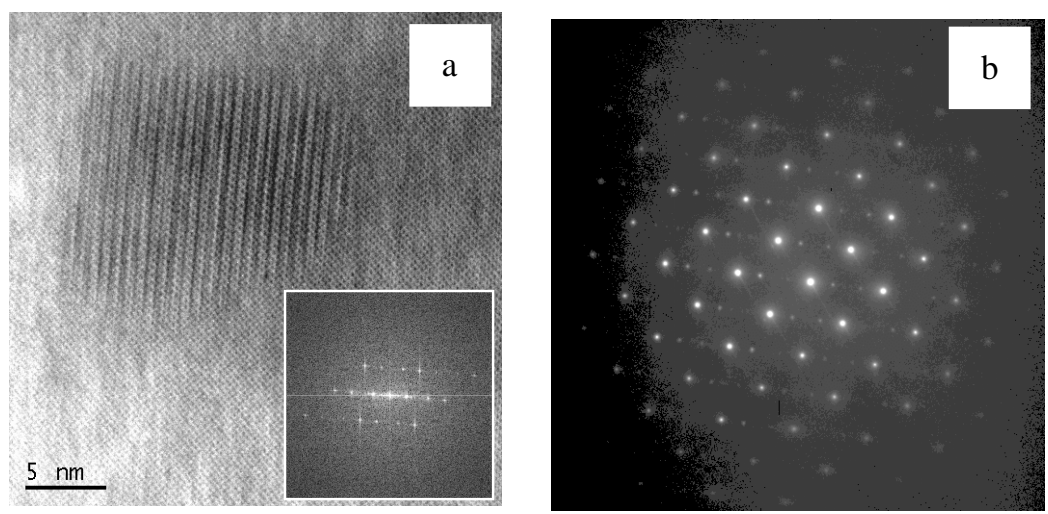

Figure 1. a) High-resolution [001] plan-view TEM image of an atomically ordered (In,Ga)Sb QD in a GaSb matrix, indicating that there is essentially no strain field associated with the atomically ordered phase. The inset Fourier transform shows double diffraction spots, i.e. the QD has a different phase to the sphalerite matrix; b) Selected area electron diffraction pattern from the same region. The images were recorded at $500{ }^{\circ} \mathrm{C}$, after the specimen was held at this temperature for 2 hours (and previously held at $475^{\circ} \mathrm{C}$ and $350{ }^{\circ} \mathrm{C}$, for 2 hours each). This suggests that the atomically ordered QD is structurally stable and that $\mathrm{T}_{\mathrm{c}}$ is of the order of magnitude of the growth temperature.
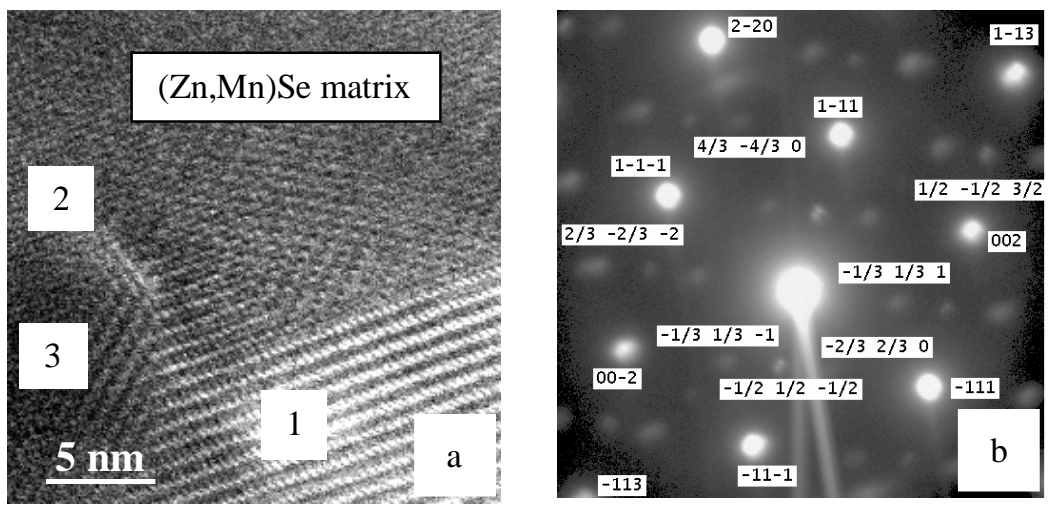

Figure 2. (a) Atomic resolution Z-contrast image, viewed in the $\langle 110\rangle$ direction, showing atomic ordering in $(\mathrm{Cd}, \mathrm{Mn}, \mathrm{Zn}) \mathrm{Se}$ agglomerates in a $(\mathrm{Mn}, \mathrm{Zn}) \mathrm{Se}$ matrix. Agglomerate 1 is actually $\sim 200 \mathrm{~nm}$ and all the larger agglomerates in this sample posses the same orientation relationship [8,9]. Although quite large, these agglomerates are usually free of structural defects, indicating that external lattice mismatch strains are negligible; (b) Selected area 
electron diffraction pattern from at least two agglomerates showing a variety of extra spots due to long range atomic ordering that indicate the existence of phases that have not been observed before in compound semiconductors [12].
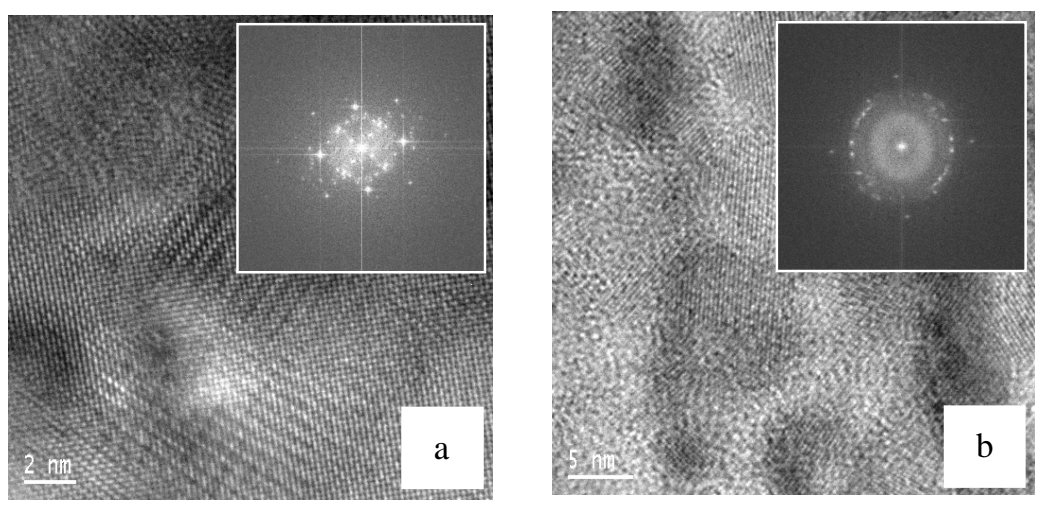

Figure 3. High-resolution TEM [001] plan-view images of atomically ordered (Cd,Zn)Se quantum dots in a $\mathrm{ZnSe}$ matrix showing long-range (a) and short-range (b) order in the same sample after 38 months storage at room temperature. The inset Fourier transforms show for (a) superlattice spots and for (b) both a diffuse and a spotty ring.

When the growth was performed at $480{ }^{\circ} \mathrm{C}$, i.e. the lowest temperature we used in our experiments [21,22], we observed for Stranski-Krastanow grown (In, Ga)Sb quantum dots in GaSb matrix, occasionally local short range order in TEM (as indicated by extra spots and diffuse to spotty rings in the diffraction patterns, images not shown) 14 days after the growth. As the time interval between growth and TEM investigations was short for these OS-QDs, we suggest that the short-range order may have formed during the growth. Since short-range order exists even above $T_{c}$ [15], we interpret these observations as further indications that structural transformations take place in this quantum dot system and that $T_{c}$ is in the order of magnitude of the growth temperature.

With the internal energies for 8 more (In,Ga)P phases ranging from 6.2 to $31.1 \mathrm{meV}$ per atom [10], and similar considerations on the entropy, it is obvious, that agglomerates with many different structures, some of which may not have been observed before in semiconductors, may originate from OS-QDs with the sphalerite structure and may co-exist in one sample in close proximity. Some of the agglomerates may have orientation relationships that lead to better lattice fits with the matrix than others and, therefore, grow over time on the expense of domains that have the larger Gibbs free energies. Such a co-existence of novel semiconductor phases has been observed experimentally, as shown in Figure 2. For StranskiKrastanow grown $(\mathrm{Cd}, \mathrm{Zn}) \mathrm{Se}$ quantum dots in a $\mathrm{ZnSe}$ matrix, we observed $\sim 38$ month after the growth, short-range and long-range order that did not appear to exist (in this amount) at the time of earlier investigations [23] (Figure 3).

Finally, we speculate on the cause for the increase of the lifetime of (In, Ga)As quantum dot lasers (to $\sim 9000$ hours from $\sim 1200$ hours) as an effect of embedding the quantum dots sheets in strain reducing (In,Ga)As layers rather than in pure GaAs spacer layers [13]. On the basis of our thermodynamic arguments, we suggest that external lattice mismatch strain driven cooperative atomic ordering (or phase separation) phenomena took place in the more severely strained QDs while the lasers were tested at room temperature. Since atomic ordering (and phase separation) reduces the bandgap [11,12], the wavelength of the emitted infrared light shifted over time to the red and the fixed $800 \mu \mathrm{m}$ cavity lengths were more and more out of tune with the lasing condition.

\section{CONCLUSIONS}

Thermodynamic arguments for the formation of atomic order in heteroepitaxially grown semiconductor quantum dots were advanced. These arguments have implications that were supported by transmission electron microscopy evidence from different III-V and II-VI compound semiconductor 
quantum dot systems. The key ingredients for the future development of a qualitative model for structural transformations in ordinarily strained quantum dots were identified.

\section{ACKNOWLEDGMENTS}

PM and TT were supported by the National Science Foundation project no. DMR-9733895. Prof. William A. Jesser from the Department of Materials Science and Engineering of the University of Virginia is thanked for bringing the seminal Bragg-Williams paper to our attention. Prof. Jacek K. Furdyna and Dr. Malgorzata Dobrowolska from the Physics Department of the University to Notre Dame are thanked for the supply of samples.

\section{REFERENCES}

1. T.P. Pearsall (editor), "Quantum Semiconductor Devices and Technologies”, (Kluwer Academic Publishers, 2000).

2. H.J. Kim and Y.H. Xie, Appl. Phys. Lett. 79, 263 (2001).

3. P.B. Joyce, P.B. Joyce and T.J. Krzyzewski, G.R. Bell, B.A. Joyce, and T.S. Jones, Phys. Rev. B 58, R15981 (1998).

4. M. Strassburg, V. Kutzer, U. W. Pohl, A. Hoffmann, I. Broser, N. N. Ledentsov, D. Bimberg, A. Rosenauer, U. Fischer, D. Gerthsen, I. L. Krestnikov, M. V. Maximov, P. S. Kop'ev, and Zh.I. Alferov, Appl. Phys. Lett. 72, 942 (1998).

5. T. Walter A.G. Cullis, D.J. Norris, and M. Hopkinson, Phys. Rev. Lett. 86, 2381 (2001).

6. P. Möck, T. Topuria, N.D. Browning, M. Dobrowolska, S. Lee, J.K. Furdyna, G.R. Booker, N.J. Mason, and R.J. Nicholas, Appl. Phys. Lett. 79, 946 (2001).

7. P. Möck, T. Topuria, N.D. Browning, L. Titova, M. Dobrowolska, S. Lee and J.K. Furdyna, J. Electron. Mater. 30, 748 (2001).

8. P. Möck, T. Topuria, N.D. Browning, G.R. Booker, N.J. Mason, R.J. Nicholas, L.V. Titova, M. Dobrowolska, S. Lee and J.K. Furdyna, Mater. Res. Soc. Symp. 640, P6.3.1 (2000).

9. T. Topuria, P. Möck, N.D. Browning, L.V. Titova, M. Dobrowolska, S. Lee and J.K. Furdyna, Mater. Res. Soc. Symp. 640, P8.3.1 (2000).

10. N. Marzari, S. de Gironcoli, and S. Baroni, Phys. Rev. Lett. 72, 4001 (1994).

11. D.B. Lanks, S-H. Wei, and A. Zunger, Phys. Rev. Lett. 69, 3766 (1992).

12. A. Zunger and S. Mahajan, "Atomic ordering and phase separation in epitaxial III-V alloys", in Handbook on Semiconductors (Elsevier Science B.V., 1994), Ed. T.S. Moss, Vol. 3, Volume Ed. S. Mahajan, pp. 1447-1514.

13. H-Y. Liu, B. Xu, Y-Q Wei, D. Ding, J-J. Qian, Q. Han, J-B Liang, and Z-G. Wang, Appl. Phys. Lett. 79, 2869 (2001).

14. W.L. Bragg and E.J. Williams, Proc. Roy. Soc. (London) A 145, 699 (1934).

15. H.A. Bethe, Proc. Roy. Soc. (London) A150, 552 (1935).

16. L.A. Girifalco and D.O. Welch, "Point Defects and Diffusion in Strained metals", (Gordon and Breach, 1967).

17. O. Madelung, ed. "Semiconductors Group IV Elements and III-V Compounds, Data in Science and Technology" (Springer, 1991)

18. Internet based semiconductor data base at http://www.ioffe.rssi.ru/SVA/NSM/Semicond/InP/thermal.html. At the date this paper was written, this URL was deemed to be useful as source of data. Neither the author nor the Materials Research Society warrants or assures liability for the content or availably of this URL.

19. A.H. Cottrell, "Theoretical Structural Metallurgy", (Edward Arnold Publ.1965).

20. W. Seifert, chapter 14 in ref. [1], pp. 139-181.

21. P. Möck, G.R. Booker, N.J. Mason, E. Alphandéry, and R.J. Nicholas, IEE Proc.-Optoelectron. 147, 209 (2000), and unpublished material.

22. E. Alphandéry, R.J. Nicholas, N.J. Mason, P. Möck, and G.R. Booker, Appl. Phys. Lett. 74, 2041 (1999).

23. C.S. Kim, M. Kim, S. Lee, J.K. Furdyna, M. Dobrowolska, H. Rho, L.M. Smith, H.E. Jackson, E.M. James, Y. Xin and N.D. Browning, Phys. Rev. Lett. 85, 1124 (2000). 